FORMATION Formation emploi

Revue française de sciences sociales

96 | octobre-décembre 2006

Quand la qualification fait débat(s)

\title{
Les jeunes jugent le travail : paroles de jeunes diplomés du baccalauréat professionnel
}

Young people's attitudes to work: the impressions of young vocational

baccalauréat holders

Fachabiturienten zum Thema Arbeit

Sophie Devineau

\section{OpenEdition}

Journals

Édition électronique

URL : http://journals.openedition.org/formationemploi/2551

DOI : 10.4000/formationemploi.2551

ISSN : 2107-0946

Éditeur

La Documentation française

Édition imprimée

Date de publication : 1 octobre 2006

Pagination : 67-79

ISSN : 0759-6340

\section{Référence électronique}

Sophie Devineau, «Les jeunes jugent le travail : paroles de jeunes diplomés du baccalauréat

professionnel », Formation emploi [En ligne], 96 | octobre-décembre 2006, mis en ligne le 15 décembre 2009, consulté le 30 octobre 2020. URL : http://journals.openedition.org/formationemploi/2551 ; DOI : https://doi.org/10.4000/formationemploi.2551 
bacheliers dans une génération, si l'enseignement général représente la moitié des diplômes $(53 \%)$, les enseignements technologique ( $28 \%$ ) et professionnel $(19 \%)$ concernent l'autre moitié. L'importance numérique de ces filières est d'autant plus remarquable que leur croissance continue sur dix ans coïncide avec un taux de baccalauréats généraux qui marque le pas. De ce point de vue, la Basse-
Normandie est une région spécifique, notamment avec un enseignement agricole et un apprentissage traditionnellement plus développés qu'ailleurs. Ainsi, en 1997, 60,1 \% d'une classe d'âge accédait en terminale générale en Basse-Normandie contre $62,7 \%$ nationalement, mais $10,9 \%$ suivaient un enseignement professionnel contre 9,6\% sur l'ensemble du territoire. On vérifie ce facteur régional particulière-

\section{Encadré 1 \\ L'enquête, I'analyse textuelle et de contenu}

\section{ENQUETE}

Enquête complémentaire de l'enquête SuBaNor96* "Les bacheliers professionnels Bas-Normands février 98 »-Organisme responsables : ORFS de Caen

Champ de l'enquête: Les diplômés du baccalauréat professionnel en 1996 de Basse-Normandie, 18 mois après leur diplôme. Effectif : 1114 diplômés en 1996.

Date de réalisation: Février 98, 2è interrogation 18 mois après la 1 ère interrogation de l'automne 96 au moment du retrait du diplôme.

Support de l'enquête: questionnaire "bacheliers professionnels».

Thèmes : - Parcours scolaire - Parcours de recherche d'un emploi - Jugements du Bac Pro -

Question ouverte n० 143 : "En définitive, qu'en pensez-vous, du baccalauréat professionnel? 》. Effectif : 902 réponses.

* Subanor96 est un suivi de cohorte de bacheliers bas-normands sur une durée de 5 ans à 10 ans. II n'y a pas d'interrogation systématique tous les ans de tous les bacheliers mais une interrogation à $T+2$ (24 mois) pour les bacheliers professionnels, à $T+4$ pour les bacs généraux avec suivi à l'université.

\section{ANALYSE}

a) Analyse textuelle : sur le corpus global des réponses des individus, comptage des mots et des cooccurrences de mots en termes de présence-absence dans des segments de discours ou unités de discours. - Analyse factorielle pour extraire des facteurs résumant l'ensemble de l'information des tableaux croisés des mots et des unités de discours. Objectif : décrire des "mondes lexicaux". Chaque réponse d'un individu peut être découpée en un, deux ou trois segments; il n'y a donc pas identité entre le nombre d'unités de discours contenues dans une classe et le nombre d'individus.

Résultats : nombre de mots retenus $=527-$ Nombre de segments de discours classés $=567$ - Classe $1=111$ unités de discours $=20 \%$. Classe $2=285$ unités de discours $=50 \%$. Classe $3=118$ unités de discours $=21 \%$ Classe $4=53$ unités de discours $=9 \%$.

AFC : les deux premiers facteurs recueillent $71 \%$ de la variance. Facteur $1=41 \%$. Facteur $2=30 \%$. Variables supplémentaires, illustratives dans l'analyse des classes de mots : Caractéristiques sociodémographiques : Sexe - Année de naissance - Série du Bac Pro - Mention du Bac Pro - BEP - CAP Redoublement - Activité Père - Activité Mère - PCS Père - PCS Mère - Emploi : mode d'obtention Situation en 96 - Situation en 98.

b) Analyse de contenu : étude des thèmes «École » et « emploi » par individu. 
ment dans le département de l'Orne où la voie professionnelle concentre quelques $25 \%$ des baccalauréats. D'une certaine façon donc, cette région constitue un laboratoire privilégié pour l'observation du baccalauréat professionnel.

Tout semble avoir été mis en œuvre pour que ce baccalauréat soit un succès, et de fait, on rencontre une tonalité enthousiaste à son propos (Alternatives Economiques, 2005). Sur le plan régional, les bulletins de l'ORFS (1999) fournissent une image également très positive de cette filière de formation. On peut lire ainsi que "La situation scolaire en lycée professionnel est plus favorable aux élèves que celle des lycées généraux et technologiques puisque seuls $10 \%$ des élèves redoublent une année de formation » ou encore que le baccalauréat professionnel est obtenu dans de bien meilleures conditions avec un nombre de mentions en augmentation. De leur côté, les enseignants expriment aussi une très grande satisfaction en évoquant une relation privilégiée entre les élèves et l'équipe pédagogique, une forte implication de tous, des élèves très matures (ORFS, 2001).

Enfin, sur le plan national, les statistiques du ministère de l'Éducation nationale sur la situation des sortants des lycées en février 2003 montrent en effet que les titulaires d'un bac pro sont $27 \%$ à être au chômage contre $33,4 \%$ des titulaires d'un CAP/BEP (certificat d'aptitude professionnelle; brevet d'études professionnelles), ou encore $49,3 \%$ de ceux n'ayant aucun diplôme. Et cinq années plus tard, parmi les titulaires d'un bac général, 11 \% sont au chômage de même que $10 \%$ de ceux qui possèdent un bac technologique contre $8 \%$ pour les bacheliers professionnels (ORFS, 2001).

Reste les jeunes et le regard qu'ils portent sur ce diplôme. Ce qu'ils expriment interroge autant l'institution scolaire et ses modèles de réussite que l'entreprise dans ses modes de recrutement des jeunes.

\section{LE RECUEIL DE LA PAROLE DES JEUNES}

Dans ce bel ensemble laudatif, où tous les acteurs du système scolaire expriment leur satisfaction, il nous a donc paru intéressant de recueillir l'avis des jeunes bacheliers du diplôme professionnel. Nous avons choisi de réaliser cette observation en BasseNormandie, à partir des 902 réponses à la question ouverte suivante : «En définitive, que pensez-vous du baccalauréat professionnel? 》 Question posée dixhuit mois après l'obtention du diplôme. Le questionnaire comportait un ensemble de réponses à des questions fermées retraçant le parcours scolaire et d'insertion. La question ouverte était proposée immédiatement après une série de questions fermées sur le même sujet ; on pouvait craindre alors un effet d'interférence biaisant les résultats. Toutefois, la comparaison avec les entretiens approfondis menés dans une enquête complémentaire à l'enquête quantitative (ORFS, 2001) montre des résultats convergents pouvant rassurer sur la qualité des réponses pour cette question d'opinion.

L'étude du texte des réponses a été réalisée selon une analyse thématique d'une part, et complétée d'autre part d'une analyse textuelle, laquelle procède au comptage des mots ou couples de mots (Jenny, 1997 ; Demazière, 2005). Un mot sera associé à d'autres mots à condition qu'ils apparaissent fréquemment en même temps. Ainsi repérés, des groupes de mots liés significativement entre eux permettent d'établir des types d'argumentations fortement présentes dans le discours global de ces jeunes. Par exemple, le mot "difficile» est souvent employé avec les mots « poursuivre », « étude », « par rapport», « BTS » (brevet de technicien supérieur), ce qui permet de repérer dans les réponses une préoccupation d'ordre scolaire. Du point de vue interprétatif, cette approche postule que les associations de mots (signifiants) ne sont pas dues au hasard, mais que cela correspond au contraire à des réalités (signifiés) vécues à travers des représentations sociales (référent). Dans l'exemple donné, certains jeunes décrivent la hiérarchie scolaire des diplômes et disent la vivre sur un mode négatif.

Préalablement à l'analyse des réponses, nous avons identifié les locuteurs selon leurs caractéristiques sociodémographiques, afin de contextualiser leurs propos. Dans cette enquête SuBaNor96, « Les bacheliers professionnels », comme ailleurs les enfants d'ouvriers ou d'employés, représentent plus de la moitié des bacheliers professionnels, et les enfants d'agriculteurs $11 \%$ à eux seuls, alors qu'ils ne sont que $4 \%$ parmi l'ensemble des jeunes de 18 à 20 ans en Basse-Normandie. Si certaines catégories sociales sont largement surreprésentées dans ces filières, ce n'est pas le cas des enseignants, dont seulement $1 \%$ des enfants suivent ce type de formation alors qu'ils sont $5 \%$ parmi l'ensemble des jeunes de 18 à 20 ans 
en Basse-Normandie. Les enfants de cadres et professions libérales sont également sous-représentés puisque seuls $8 \%$ sont dans la voie professionnelle quand ils comptent pour $12 \%$ des jeunes de 18 à 20 ans en Basse-Normandie. Ensuite, ces jeunes ont un parcours scolaire marqué par des redoublements, six élèves sur dix ont deux ans ou plus de retard. Les profils sociologiques, familiaux et scolaires de ces jeunes pourront éclairer le jugement qu'ils portent sur leur formation professionnelle et la place qu'ils accordent au monde du travail.

Dans un premier temps, nous serons attentifs à la structure de leurs discours, aux mots saillants autour desquels les jeunes argumentent leurs opinions. Nous nous appuierons pour cela sur les résultats de l'analyse des mots. Dans un deuxième temps, on analysera plus spécifiquement chacun des thèmes apparus comme importants : d'abord l'aspect éducatif de leur formation puis la relation à l'emploi.

\section{LE TRAVAIL, UNE VALEUR CENTRALE DU DISCOURS}

Le travail fonctionne comme la valeur centrale du discours de ces bacheliers, aussi bien lorsqu'ils évoquent le travail scolaire ou l'emploi en entreprise.
Les réponses des jeunes s'organisent selon deux thématiques. La première décrit la valeur d'usage du diplôme. Les propos, d'une tonalité généralement positive, insistent sur l'aspect initiatique à la vie adulte de ces formations. Parmi les attributs positifs, on relève également le caractère réparateur d'un échec scolaire antérieur, l'aspect concret et directement lié à la vie active, «la vraie vie ».

$\mathrm{La}$ deuxième thématique interroge la valeur d'échange du diplôme. Le manque de reconnaissance du diplôme sur le marché du travail comme au sein de l'institution scolaire est ici discuté par les jeunes. La faible valeur du diplôme professionnel à l'épreuve de la hiérarchie des salaires ou de la hiérarchie scolaire suscite un sentiment d'amertume.

Entre ces deux pôles, valeur formatrice positive du bac pro d'un côté et valeur marchande négative de l'autre, on peut observer des variations selon le registre qui va être privilégié, l'école ou l'emploi. Par exemple, sur le versant de l'emploi, le bac pro est tantôt jugé négativement à travers les choix de branches ou de secteurs professionnels, tantôt positivement à travers les stages en entreprise, premiers pas vers l'emploi. Sur le versant scolaire, la formation va être appréciée tantôt positivement par ses contenus adaptés à la vie active, tantôt négativement par la comparaison défavorable du bac pro au BTS.

\section{Encadré 2 \\ Classe $\mathrm{n}^{\circ} 1$ : Le bac pro : I'utilité scolaire du diplôme}

Phrase type : "Très bon diplôme qui prépare bien à la vie active ".

Les jugements positifs sont très majoritaires dans cette classe.

Ce groupe lexical représente $20 \%$ des unités de discours analysées.

Phrases illustratives de la classe :

"-Prépare l'entrée dans la vie active.

- Formation complète permettant d'entrer plus facilement dans la vie.

- C'est le minimum pour entrer dans la vie active.

- Très bon diplôme qui prépare bien à la vie active.

- Le baccalauréat professionnel est une bonne formation pour rentrer rapidement dans la vie active.

- Le baccalauréat professionnel est une bonne formation dans le but d'entrer dans la vie professionnelle ».

Variables corrélées $\left(\square^{2}\right)$ à cette classe lexicale :

- Bac Bâtiment Métal Alu - Mère agricultrice - Né en 75 - 


\section{Encadré 3}

\section{Classe $n^{\circ} 4$ : Le bac pro et l'expérience : l'intérêt des stages}

Phrase type : "Permet d'acquérir une expérience professionnelle grâce aux stages 》.

Les jugements positifs sont très majoritaires dans cette classe.

Ce groupe lexical représente $9 \%$ des unités de discours analysées.

Phrases illustratives de la classe :

«-Permet d'acquérir une expérience professionnelle grâce aux stages, permet d'acquérir une qualification.

- Le baccalauréat professionnel permet d'acquérir des connaissances pratiques et théoriques. II permet d'avoir une expérience grâce aux nombreux stages.

- Cela permet d'apprendre des matières professionnelles, connaître le monde du travail grâce aux différents stages. Durée du stage trop court.

- Il nous permet aussi d'apprendre un métier par le biais de différents stages de formations professionnelles en entreprise.

- Permet d'avoir une expérience professionnelle. Permet de rencontrer des gens et peut-être trouver son premier emploi grâce à un stage effectué ».

Variable corrélée $\left(\square^{2}\right)$ à cette classe lexicale :

- Bac Productique Mécanique

Ainsi, le croisement de ces deux axes, Valeur d'échange (négatif) /Valeur d'usage (positif) d'une part, et d'autre part École/Emploi décrit quatre types de discours.

La classe 1 est celle d'un discours en général positif, centré sur l'intérêt scolaire de la formation. Dans ce groupe lexical, le jugement porté sur le bac pro se fonde d'abord sur le modèle scolaire comme propédeutique à la vie. Les discours mettent en avant l'utilité du diplôme (soit la portée initiatique à la vie adulte) en termes d'accès à la vie active, avec des appréciations en majorité positives. C'est bien la vie active qui explique l'intérêt du bac pro puisque cette formation intègre cet aspect dans les cours. Il y a donc une dimension évaluative de l'enseignement concret contre l'enseignement abstrait dans lequel ils ont pu connaitre des échecs scolaires. On retrouve là "l'anti-intellectualisme » populaire décrit par Gilles Moreau (2003).

La classe 4 est celle d'un discours également plutôt positif, mais l'intérêt des stages est ici central. Dans ce groupe lexical, le bac pro est évalué à l'aune des emplois et de l'ouverture de l'École sur le marché du travail. Les stages en entreprises, organisés pendant la formation, sont très appréciés par le contact direct qu'ils ménagent avec la réalité du monde du travail et par le sentiment qu'ils donnent d'une expérience professionnelle.

La classe 2 véhicule un discours majoritairement négatif cette fois. Le bac pro est jugé dans une perspective de poursuite d'études. Dans ce groupe lexical, les jeunes abordent le problème de la faible valeur scolaire du bac pro en comparaison des autres bacs, technologiques ou généraux, quant aux chances de poursuivre des études, notamment de préparer un BTS. Dans la hiérarchie des diplômes, la mention professionnelle a détruit le prestige qui auréolait le titre de bachelier, comme le soulignait Stéphane Beaud dans $80 \%$ au bac, et après? (2002).

La classe 3 est également porteuse d'un discours plutôt négatif qui confronte le bac pro à l'emploi. Ici, les discours sont centrés sur la relation diplôme / marché de l'emploi, soit l'utilité du diplôme en termes de carrières professionnelles, de projet professionnel dans une spécialité, un secteur ou une branche d'activité et non plus seulement en termes généraux d'entrée dans la vie active. Les appréciations sont marquées par l'amertume, le regret, la déception. 


\section{Encadré 4}

\section{Classe $\mathrm{n}^{\circ} 2$ : Le bac pro et les études : la faible valeur scolaire du diplôme}

Phrase type: "Comparé au BTS le bac pro n'a qu'une très faible valeur 》.

Les jugements négatifs sont très majoritaires dans cette classe.

Ce groupe lexical représente $50 \%$ des unités de discours analysées.

Phrases illustratives de la classe :

" - Il faudrait poursuivre en BTS, de préférence en alternance, mais les bac pro ne sont malheureusement pas prioritaires.

- Il faudrait peut-être faire en sorte que le niveau du bac pro soit plus élevé pour permettre une bonne poursuite d'études en BTS.

- La poursuite en BTS est bien mais le niveau de vente n'est pas du tout une continuité mais un retour à zéro lorsque l'on vient d'un bac pro.

- Le baccalauréat professionnel permet de connaître le milieu professionnel. Il est conseillé à ceux qui désirent arrêter leurs études après le bac mais pas à ceux qui souhaitent continuer les études.

- Comparé au BTS, le bac pro n'a qu'une très faible valeur. "

Variables corrélées $\left(\square^{2}\right)$ à cette classe lexicale :

- Bac Maintenance Réseaux Bureautique Télématique - Bac Structures Métalliques - Mention AB - Mère enseignante - Né en 73.

La vie active, dans sa dimension non plus scolaire, mais professionnelle, paraît ici nettement moins réjouissante. À l'épreuve de la hiérarchie des salaires, le bac pro leur semble correspondre aux plus bas salaires.

Chacun de ces argumentaires, plus ou moins présents dans les réponses de chaque individu, renvoie à des profils sociologiques repérés à travers quelques variables sociodémographiques. Parmi les discours positifs, on relèvera pour la classe 1 que ce sont des enfants d'agricultrices, ainsi que des jeunes nés en 1975, ou bien des titulaires d'un bac «Bâtiment Métal », qui apprécient le modèle scolaire de leur formation. Dans ce groupe, on a probablement affaire à des jeunes qui ne souhaitaient pas rester de longues années à l'École. Pour la classe 4, le discours n'est associé qu'à une caractéristique, la spécialité du bac «Productique Mécanique ». Ce sont les jeunes de ces filières qui mettent particulièrement en avant l'intérêt des stages dans leur formation :

"-Très bonne complémentarité, le juste milieu entre la théorie et la pratique. Apprentissage d'une méthodologie et donc adaptation à un grand nombre de situations en autonomie. Application directe de la théorie dans la vie active plus l'expérience. "
(Homme, né avant 1973, Bac Structures Métalliques, Mention assez bien, BEP : non ; CAP : oui ; Redoublement : oui ; Père : actif, Mère : active ; Père : artisan, Mère: employée; Mode d'obtention de l'emploi : autre; Situation en 96 : autre ; Situation en 98 : travail). «-C'est une bonne filière car nous avons plus de chance sur le marché de l'emploi par rapport à ceux qui font un baccalauréat général, grâce aux stages nous avons un peu d'expérience. »

(Homme, né en 1976, Bac : Productique Mécanique, Mention passable ; BEP : oui ; CAP : oui ; Redoublement : oui ; Père : actif; Mère : active ; Père : employé ; Mère : ouvrière; Mode d'obtention de l'emploi : seul ; Situation en 96 : chômage ; Situation en 98 : travail) ».

Parmi les discours négatifs, pour la classe 2, ce sont des enfants d'enseignants qui évoquent le plus le problème de la poursuite des d'études. Les spécialités de ces jeunes sont la « Maintenance Réseaux Bureautique Télématique » ou encore les « Structures Métalliques ». Ils ont eu leur bac pro avec la mention assez bien et sont nés en 1973. Dans la classe 3, les regrets concernant le choix de la branche et la déception vécue sur le marché de l'emploi sont caractéristiques 


\section{Encadré 5 \\ Classe $n^{\circ} 3$ : Le bac pro et le type d'emplois : la faible valeur du diplôme sur le marché du travail}

Phrase type : "J'ai plutôt été dégradé qu'autre chose. "

Les jugements négatifs sont très majoritaires dans cette classe.

Ce groupe lexical représente $21 \%$ des unités de discours analysées.

Phrases illustratives de la classe :

"-Je pensais que cela me servirait dans mon parcours professionnel. J'ai plutôt été dégradé qu'autre chose.

- Cela ne m'a rien apporté puisque que je n'ai pas trouvé de travail dans cette branche.

- Je suis contente de l'avoir eu. Mais il ne me sert à rien sinon j'aurais travaillé dans un lieu me convenant plus.

- Aujourd'hui, je me bats car j'ai toujours un coefficient de BEP et mon employeur tire profit de mes connaissances sans tenir compte de ma rémunération.

- Si j'avais su, je n'aurais jamais entrepris ces cours, j'ai vraiment tout perdu. »

Variables corrélées $\left(\square^{2}\right)$ à cette classe lexicale :

- Bac Vente - bac Construction Bâtiment Gros OEuvre - Mention B - Père chômeur - Né avant 66 - Né entre 66 et 72.

de jeunes titulaires d'un bac pro « Vente » ou « Construction Bâtiment Gros CEuvre » ou dont le père est chômeur. Ils ont obtenu leur diplôme avec une mention bien et sont plus âgés que les autres puisqu'ils sont nés avant 1966 ou entre 1966 et 1972.

Ainsi, ce sont les enfants d'enseignants en mobilité familiale descendante ou les enfants de chômeurs taraudés par la question de l'emploi qui éprouvent le plus de réserves :

«- La poursuite en BTS est bien mais le niveau de vente n'est pas du tout une continuité mais un retour à zéro lorsque l'on vient d'un baccalauréat professionnel. »

(Femme, née en 1976 ; Bac Vente, Mention assez bien ; BEP : oui ; CAP : non; Redoublement : oui ; Père : actif ; Mère : active ; Père: agriculteur ; Mère : enseignante; Mode d'obtention de l'emploi : non réponse ; Situation en 96 : études ; Situation en 98 : études).

«-Le baccalauréat professionnel est une bonne chose. Il permet d'avoir une petite expérience professionnel mais n'est pas suffisant pour trouver un emploi ».

(Homme, né en 1974, Bac Maintenance Réseau Bureautique Télématique, Mention passable; BEP
Oui ; CAP : non ; Redoublement : oui ; Père : actif ; Mère : active ; Père : ouvrier ; Mère : agricultrice ; Mode d'obtention de l'emploi : non réponse ; Situation en 96 : études; Situation en 98 : études).

«-Le baccalauréat professionnel est un diplôme que les employeurs apprécient. Dommage que l'on ferme les portes pour les BTS. Il serait intéressant de mettre une classe intermédiaire entre le baccalauréat et le BTS.»

(Homme, né en 1974; Bac Structures Métalliques, Mention passable ; BEP : non; CAP : non ; Redoublement: oui ; Père: actif; Mère: non réponse ; Père: profession libérale; Mère: non réponse; Mode d'obtention de l'emploi : seul : Situation en 96 : autre ; Situation en 98 : travail)

«- Enseignement très intéressant et nous sortons avec une formation. Il est très peu reconnu dans le domaine du secrétariat. Il est toujours demandé le niveau BTS. Il est très difficile d'entreprendre des études supérieures après le baccalauréat professionnel car nous devons entrer dans la vie active. "

(Femme, née en 1977; Bac Secrétariat, Mention assez bien ; BEP : non ; CAP : non : Redoublement : non; Père : chômeur; Mère : inactive; Père : 
ouvrier; Mère : non réponse; Mode d'obtention de l'emploi : non réponse; Situation en 96 : études; Situation en 98 : chômage).

«-Je suis contente de l'avoir eu. Mais il ne me sert à rien sinon j'aurai travaillé dans un lieu me convenant plus. "

(Femme, née en 1975 ; Bac Vente, Mention passable ; BEP : oui ; CAP : non; Redoublement : oui ; Père : chômeur ; Mère : inactive ; Père : ouvrier; Mère : non réponse; Mode d'obtention de l'emploi : non réponse; Situation en 96 : chômage; Situation en 98 : autre).

L'analyse textuelle traitant essentiellement de liaisons entre des mots contenus dans les réponses, on n'interprètera pas le volume des classes en nombre d'individus. Répondant souvent sous la forme «le bac pro c'est un bon diplôme, mais il n'est pas assez reconnu », la majorité des jeunes est concernée par les aspects de jugement positif des classes 1 et 4 (respectivement $20 \%$ et $9 \%$ du corpus), ou plus négatifs des classes 2 et 3 (respectivement $50 \%$ et $21 \%$ ). Au total, on s'aperçoit que les jeunes ont orienté leurs réponses vers la question de l'emploi ou de la poursuite d'études, davantage que vers leur formation à l'École. Cela renvoie en partie à leurs préoccupations au moment de l'interrogation; dix huit mois après l'obtention du diplôme, c'est la valorisation de cet acquis qui focalise leur attention. L'ensemble des discours étant fortement structuré par la question de l'utilité du diplôme, on constate que les réponses sont tirées vers des argumentations critiques de la part des jeunes. Pour autant, il n'est pas anodin pour l'évaluation de l'École de noter qu'elle concentre les opinions positives.

\section{UNE FORMATION SUR MESURE}

L'analyse du vocabulaire permet de mettre en évidence une École sur mesure selon ces jeunes. Ils déclarent généralement avoir aimé cet enseignement, les jugements négatifs n'intervenant qu'au moment de la préparation d'un BTS ou de l'entrée en emploi.

Dans ce registre, le relevé des thèmes développés par les jeunes montre que toutes les valeurs positives associées à l'École sont liées au travail scolaire et professionnel. Les oppositions telles que «concret / abstrait»; «pratique / théorique »; « vie active / vie passive »; « doué / non doué », sont chaque fois mobilisées en faveur de la figure emblématique de l'activité professionnelle. La vraie vie, la vie concrète et active n'est concevable pour ces jeunes qu'au travers de l'emploi. Le modèle scolaire classique est renversé au profit du monde du travail, et l'effort dans les études vise un métier, un salaire. À l'opposé de la position infantilisante de l'élève, le bac pro consacre un statut d'adulte parce qu'il confère de l'autonomie et permet les initiatives. L'un deux dira :

"Convient aux lycéens qui aiment prendre des initiatives et qui aiment la vie. »

(Femme, née en 1976; Bac Commerce; Mention passable ; BEP : oui ; CAP : non; Redoublement : oui ; Père : actif ; Mère : active ; Père : artisan ; Mère : employée; Mode d'obtention de l'emploi : seul; Situation en 96 : travail ; Situation en 98 : études).

\section{* Exemples tirés des phrases illustratives des classes :}

«-Le baccalauréat professionnel prépare bien à la vie active. (Classe 1)

- Formation complète permettant d'entrer plus facilement dans la vie. (Classe 1)

- On a autant de stages professionnels que de théorie. Les stages nous montrent la réalité de la vie active. (Classe 1)

- Convient aux lycéens qui aiment prendre des initiatives et la vie. (Classe 1)

- Les stages apportent une plus grande maturité. (Classe 1)

- Permet de débattre sur des sujets de la vie professionnelle. Le baccalauréat professionnel est une vraie réussite. (Classe 1)

- Le baccalauréat professionnel est un bon niveau scolaire pour entrer dans la vie active. Il manque toutefois un peu de pratique: stages et atelier. (Classe 1)

- Le baccalauréat professionnel m'a permis de me sortir du monde scolaire. (Classe 3)

- Si j'ai choisi la branche professionnelle c'est pour plus de sécurité. (Classe 3). »

Il apparaît ensuite que l'usage de ces catégories reprend en partie le discours ambiant qui prévaut pour décrire à la fois des échecs scolaires - les élèves sont non doués - et des solutions à y apporter - il leur faut un enseignement adapté et donc pratique. On peut au moins observer à quel point ces jeunes ont intériorisé les formes du jugement qui distinguent l'intelligence 
concrète de l'intelligence abstraite, comme l'exprime cette réponse à la question «En définitive, que pensez-vous du bac pro?» :

«c'est mieux quand on aime se servir de ses mains et pas que de sa tête"

(Homme, né en 1976 ; Bac Artisanat et Métiers d'art, communication graphique, Mention passable ; BEP : oui ; CAP : non ; Redoublement : oui ; Père : actif ; Mère : active ; Père : ouvrier ; Mère : ouvrière ; Mode d'obtention de l'emploi ; autre ; Situation en 96 : chômage ; Situation en 98 : travail).

\section{* Exemples tirés des phrases illustratives des classes :}

"-Le baccalauréat professionnel est une opportunité même si on ne trouve pas l'emploi idéal du premier coup, correspond à mes capacités. (Classe 3)

- Le baccalauréat professionnel est une chance pour les jeunes qui ne peuvent pas faire trop d'études. (Classe 2)

- Il peut être une solution pour les élèves qui ne sont pas bons à l'école. Les jeunes qui doivent choisir leur métier n'ont pas beaucoup d'informations, il faudrait revoir l'orientation. (Classe 2)

- Lorsque l'on n'est pas très doué en matière générale cela aide. Difficile de poursuivre des études après un baccalauréat professionnel. (Classe 2)

- Pas de regrets, quand on continue les matières sont difficiles à surmonter. Le BTS par alternance est la meilleure façon de continuer des études pour un baccalauréat professionnel ». (Classe 2)

Il en déduisent qu'ils n'ont pas de valeur intellectuelle et qu'il n'y a pas de lien avec la vie active dans les cursus généraux, dans le sens où la vie véritable est rejetée hors d'une École dont les enseignements déréalisent les contenus. On touche ici à l'obstacle majeur rencontré dans la filière générale par les jeunes issus des milieux défavorisés, celui d'une acculturation trop radicalement distante de leurs expériences de vie. L'enseignement professionnel répond effectivement à des besoins d'un autre type de réussite scolaire chez ces jeunes. Pourtant, nombreux aussi sont ceux qui réclament plus d'enseignement général afin de pouvoir poursuivre des études.

L'enseignement professionnel, en remotivant les élèves, leur a non seulement permis d'obtenir un diplôme mais les a conduits à être demandeurs de plus de formation générale. Ainsi, en retrouvant une réussite scolaire, ces jeunes ont pu se réconcilier avec le projet d'étudier. Certains jeunes évoquent clairement la réparation psychologique :

"- Le baccalauréat professionnel est une filière très intéressante pour les personnes en doute de leurs capacités. »

(Homme, né en 1977, Bac Équipement Installation Électrique, Mention bien; BEP : oui ; CAP : non ; Redoublement : non; Père : actif; Mère : active ; Père : artisan; Mère : profession libérale; Mode d'obtention de l'emploi : non réponse ; Situation en 96 : études ; Situation en 98 : alternance)

" Formation intéressante pour les jeunes qui ont perdu confiance dans le système scolaire traditionnel. Permet de prendre contact avec le monde professionnel. Permet de prendre de l'assurance en soi. »

(Sexe : non réponse ; né : non réponse ; Bac Artisanat et Métiers d'art, Communication graphique; Mention assez bien; BEP : non ; Cap : oui ; Redoublement : oui ; Père : actif; Mère : inactive ; Père : profession libérale ; Mère : non réponse ; Mode d'obtention de l'emploi : seul; Situation en 96 : travail ; Situation en 98 : travail)

«-Les stages m'ont permis d'affermir ma personnalité. »

(Femme, née en 1974; Bac Secrétariat, Mention passable ; BEP oui ; CAP : oui ; Redoublement : oui ; Père : non réponse; Mère: active ; Père : non réponse; Mère: ouvrière; Mode d'obtention de l'emploi : non réponse; Situation en 96 : études; Situation en 98 : alternance).

Il s'agit là d'un aspect non négligeable de cet enseignement; s'il est parfois contesté quant à sa valeur d'échange dans la vie active, celui-ci prend par sa dimension éducative un aspect exemplaire dans l'École cette fois. Il est ainsi remarquable, que, sur 902 réponses, aucun jeune n'ait employé le mot «échec ».

* Exemples tirés des phrases illustratives des classes :

"-Bien dans l'ensemble, il prépare très bien à la vie active, il faudrait ajouter toutefois un peu plus de matières générales. (Classe 1)

- Difficile de poursuivre des études surtout à cause des matières générales. (Classe 1)

- Il paraît que le programme de mathématiques en baccalauréat professionnel MSMA (maintenance des systèmes mécaniques automatisés) va être 
allégé, ce qui est une erreur à ne pas faire, il faudrait au contraire aller plus loin. (Classe 2)

- Après le baccalauréat, il devrait y avoir des études supplémentaires obligatoires avant d'entrer dans la vie active. (Classe 1)

- Il est très difficile d'entreprendre des études supérieures après le Bac professionnel. (Classe 1)

- Il faudrait peut-être faire en sorte que le niveau du baccalauréat professionnel soit plus élevé pour permettre une bonne poursuite d'études en BTS. (Classe 2)

- Pas le niveau pour rentrer en BTS, obligation de faire une remise à niveau. Il faut augmenter le niveau du baccalauréat professionnel (Classe 2)

- Mais on est vraiment délaissé par rapport au baccalauréat général pour accéder au BTS. Classe 2)

- Bonne formation, l'accès en BTS est difficile après un baccalauréat professionnel ». (Classe 2)

Les ombres à ce tableau scolaire sont peu nombreuses puisqu'elles n'apparaissent pas comme significatives des mots analysés. Par ailleurs, le relevé de chacune des réponses montre que seuls 18 jeunes sur les 902 critiquent les enseignants, généralement appréciés comme étant «attentifs et réceptifs aux problèmes des écoliers». Parmi les reproches qui leur sont adressés, il y a l'incompétence devant la réalité des situations de travail; de même, certains enseignants leur paraissent dépassés par les évolutions technologiques. Parfois, les jeunes mettent en cause la responsabilité des professeurs dans une orientation par défaut dans une voie de relégation, ou plus grave le mensonge des enseignants sur les promesses d'avenir de ces formations.

Cependant, pour la majorité des cas, il ressort que ce diplôme est une réussite éducative auprès des élèves. Dans le triptyque, travail-formation-emploi, le récit que font ces jeunes à l'entrée sur le marché du travail, dresse un bilan positif de l'école. C'est autour de la valeur travail comme propédeutique à l'emploi que se cristallisent tous les éloges et que se fonde la légitimité de la filière professionnelle.

\section{À CONDITION DE TROUVER UN EMPLOI RAPIDEMENT}

Le bac pro est un diplôme intéressant s'il permet au jeune de trouver un emploi rapidement. L'engage- ment de ces élèves doit ainsi être compris du point de vue de l'efficacité. Dans leurs discours, le principe d'utilité est essentiel pour décrire le bac pro, leurs projets d'études ou professionnels. Ils conçoivent leur formation à travers les métiers, la vie active, et c'est grâce à cela qu'ils adhèrent à l'enseignement professionnel.

"-Il nous permet aussi d'apprendre un métier par le biais de différents stages de formation professionnelle en entreprise. "

(Femme née en 1973; Bac Secrétariat; Mention passable; BEP : non; CAP : non; Redoublement oui ; Père : actif ; Mère : inactive; Père : ouvrier ; Mère : non réponse; Mode d'obtention de l'emploi : non réponse ; Situation en 96 : études; Situation en 98 : chômage).

«- Surtout à bien se préparer à l'emploi en faisant des stages. »

(Femme née en 1975 ; Bac Comptabilité, Mention passable; BEP : oui ; CAP : oui ; Redoublement : oui ; Père : retraité; Mère : inactive; Père : non réponse ; Mère : non réponse; Mode d'obtention de l'emploi : non réponse ; Situation en 96 : chômage ; Situation en 98 : chômage).

L'accès rapide à l'emploi est donc fondamental dans le jugement que portent ces jeunes sur leur diplôme. Or, malgré des statistiques de chômage assez flatteuses, certains jeunes, près d'un sur dix, se retrouvent sans emploi. C'est ainsi qu'il faut comprendre le bilan négatif que ceux-là dressent quand ils déclarent le bac pro inutile ou estiment avoir tout perdu lorsque l'emploi rapide promis n'est pas au rendez-vous. Ils ressentent alors les carences de ce bac soumis à la concurrence des diplômes plus élevés, mais aussi à la barrière de la sélection scolaire. Comme l'illustrent les propos d'un jeune :

«L'Éducation nationale ne donne pas la chance à certains écoliers de baccalauréat professionnel de poursuivre leurs études. Ayant un bon dossier, j'effectue mon BTS dans un lycée privé. Les lycées publics ont refusé ma candidature. »

(Femme née en 1974; Bac Vente, Mention assez bien; BEP : oui ; CAP : oui ; Redoublement : oui ; Père actif; Mère : active; Père : employé ; Mère : employée; Mode d'obtention de l'emploi : non réponse; Situation en 96 : études ; Situation en 98 : études). 
* Exemples tirés des phrases illustratives des classes :

"-Permet de rentrer assez facilement dans la vie active mais le niveau d'études atteint n'est pas suffisant. (Classe 1)

- Pas assez suffisant aujourd'hui où la sélection s'opère à bac plus deux (Classe 1)

- Certains baccalauréats professionnels offrent peu de débouchés, par exemple le secrétariat. (Classe 1)

- Si j'avais su, je n'aurais jamais entrepris ces cours, j'ai vraiment tout perdu. (Classe 1)

- Prépare bien pour travailler dés la sortie de la scolarité, à condition de trouver un emploi aussitôt, car au bout de presque 2 ans je pense que j'ai tout perdu. (Classe 3)

- Le baccalauréat professionnel n'est pas reconnu à sa juste valeur sur le marché de l'emploi.» (Classe 1)

La sociologie de l'École a mis à jour finement les mécanismes de la reproduction sociale à travers le fonctionnement d'une orientation scolaire par l'échec (M. Duru-Bellat, 1988 ; P. Merle, 2002). L'expérience scolaire de l'indignité est douloureuse et peut couper l'élève de l'envie d'apprendre. À défaut de poursuivre dans la voie générale, les jeunes sont alors orientés vers les filières professionnelles ou technologiques. Ainsi, les jeunes abordent cet enseignement par dépit et sans grande motivation. Or, leur récit révèle le retournement positif de leur rapport à la formation, au travail scolaire via une adhésion forte à la valeur travail dans la société. Ils ont pris acte de leurs faiblesses dans les performances académiques, et ils abordent le bac pro avec un réalisme modeste. Il y mettent cependant une condition, la contrepartie d'études courtes doit être l'accès rapide à un emploi. Lorsque ce contrat explicite dans l'intitulé du bac pro est rompu par l'expérience du chômage, alors le sentiment d'avoir été dupé surgit. La confrontation infructueuse avec le marché du travail induit l'idée d'une injustice sociale.

Lorsqu'ils déclarent avoir tout perdu au jeu scolaire, c'est qu'ils ont réussi au bac pro mais qu'il n'y a pas d'emploi à la clé. Les jugements négatifs interviennent a posteriori. Plus exactement, c'est l'environnement de cette formation qui est critiqué : l'usage social qui lui est réservé. Ils leur semble que la valeur d'échange du diplôme est inférieure à celle d'autres diplômes concurrents : moindre pour l'accès à une formation que celle des bacs généraux, elle serait également insuffisante en regard des BTS sur le marché de l'emploi.

En somme, ces jeunes estiment avoir été trompés s'ils perdent le bénéfice d'une insertion rapide, particulièrement pour ceux d'entre eux qui ont dû renoncer à des études longues. L'expression de leur amertume, de leur déception tranche ici avec l'enthousiasme qu'ils affichent devant l'expérience professionnelle, la vie active, les stages. Quand il n'y a pas d'emploi au bout, ils semblent ne plus être en mesure d'assumer leur diplôme. Une jeune femme témoigne ainsi du succès sémantique d'un titre scolaire emblématique, le baccalauréat:

«-Mais je suis contente d'en être arrivée jusqu'au baccalauréat et de l'avoir obtenu. »

(Femme née en 1974 ; Bac Comptabilité, Mention passable ; BEP : oui ; CAP : oui ; Redoublement : oui ; Père : actif ; Mère : active ; Père : non réponse ; Mère: employée ; Mode d'obtention de l'emploi : stage; Situation en 96: travail; Situation en 98 : travail).

Très vite, l'usage du titre de bachelier ne suffit pas à satisfaire les jeunes, qui, une fois en entreprise, ont le sentiment amer de n'être pas considérés.

«-Il y a trop de baccalauréats professionnels en circulation, maintenant on nous solde "

(sexe : non réponse; né : non réponse ; Bac Équipement Installation Électrique : installation, Mention assez bien ; BEP : non ; CAP : oui ; Redoublement : oui ; Père : retraité ; Mère : retraitée ; Père : agriculteur ; Mère : agricultrice ; Mode d'obtention de l'emploi : autre ; Situation en 96 : travail ; Situation en 98 : travail).

"-C'est un enseignement de qualité mais qui ne sert à rien car on ne peut pas trouver un emploi. »

(Femme née avant 1973; Bac Secrétariat, Mention passable; BEP : oui ; CAP : oui ; Redoublement : oui ; Père : actif; Mère : active ; Père : ouvrier ; Mère : ouvrière ; Mode d'obtention de l'emploi : non réponse; Situation en 96 : chômage; Situation en 98 : chômage).

"-Ne devrait plus exister car il n'est pas du tout reconnu même par l'ANPE. "

(Femme née en 1976 ; Bac Comptabilité, Mention : non réponse ; $\mathrm{BEP}$ : oui ; CAP : oui ; Redoublement : 
oui ; Père : actif ; Mère : chômage ; Père : ouvrier ; Mère: ouvrière; Mode d'obtention de l'emploi : ANPE ; Situation en 96 : chômage ; Situation en 98 : alternance).

La déception est à la mesure de la force du symbole, le baccalauréat restant le passeport pour le supérieur. Ces jeunes d'origine défavorisée réalisent l'importance de la mention qui accompagne le diplôme « général, technologique ou professionnel ». Tout le discours des bacheliers professionnels exprime ce balancement entre la fierté d'avoir réussi quelque chose, le baccalauréat, et le sentiment de ne pas recevoir la reconnaissance sociale attendue.

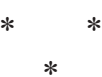

Dans l'ensemble, les résultats confortent les études précédentes, à savoir que le bac pro prépare à la vie active mais n'ouvre pas de perspectives de poursuites d'études et qu'il peut être fortement concurrencé sur le marché du travail. En revanche, la parole de ces jeunes décrit la façon dont ils déchantent quand ils en prennent conscience. Et cela s'inscrit dans la forme de leurs réponses construites souvent en deux parties, la première, positive, suivie d'une deuxième marquant des réserves. Parfois, quelques caractéristiques individuelles des jeunes ou le secteur d'activité éclairent les regrets à propos de l'accès à l'emploi et aux études. Il ressort de l'analyse du vocabulaire une structuration forte des réponses autour du thème de l'emploi à travers les mots «branche», «pratique », « stages », « entreprise » et « qualification». L'engagement dans une formation pour un emploi décrit l'École idéale où le travail scolaire est étroitement lié au travail professionnel. La vie active focalise tout le discours de ces jeunes et organise a posteriori les jugements sur ce diplôme. Partie prenante du changement opéré par l'École, alors même que la réussite pédagogique est au rendez-vous, ils ne sont plus certains d'avoir fait le bon choix. S'il n'y a pas la récompense d'un emploi rapide, le contrat semble rompu, activant un sentiment d'injustice. On est en tout cas devant un exemple où le succès éducatif peut être sans effet sur la difficulté de l'insertion, montrant ainsi les limites des innovations scolaires. Autrement dit, le baccalauréat professionnel a le mérite d'exister et de satisfaire les élèves, mais il ne saurait résoudre à lui seul la difficile question de la relation formation-emploi. 


\section{Bibliographie}

Beaud S. (2002), 80\% de réussite au bac... Et après?, Les enfants de la démocratisation scolaire, Paris, La Découverte

Demazière D. (2005), «Des logiciels d'analyse textuelle au service de l'imagination sociologique », Bulletin de Méthodologie Sociologique, janvier, 85, pp. 5-9.

Duru-Bellat M. (1988), Le fonctionnement de l'orientation. Genèse des inégalités sociales à l'école, Genève-Paris, Delachaux \& Niestlé.

Jenny J. (1997), « Méthodes et pratiques formalisées d'analyse de contenu et de discours dans la recherche sociologique française contemporaine. Etat des lieux et essai de classification ", Bulletin de Méthodologie Sociologique, 54, janvier, pp. 64-122.

Merle P. (2002), La démocratisation de l'enseignement, coll. « Repères », Paris, La Découverte.
Moreau G. (2003), Le Monde Apprenti », La Dispute.

ONISEP-Céreq (2005), «De l'école à l'emploi », Alternatives économiques, Hors série Pratique, $\mathrm{n}^{\circ} 17$, janvier.

ORFS (1999), Bacheliers professionnels. Parcours et devenirs professionnels des diplômés bas-normands (promotions 1994 / 1995 / 1996), janvier.

ORFS (2001), Le baccalauréat professionnel. Quelques représentations autour de la formation et de l'insertion professionnelle, mai.

Roussiau N., Le Blanc A. (2001), «Représentations sociales du travail et formations scolaires ou professionnelles des lycéens: approche comparative », L'Orientation Scolaire et Professionnelle, vol. XXX, $\mathrm{n}^{\circ} 1$, mars.

\section{Résumé}

\section{Les jeunes jugent le travail : paroles de jeunes diplômés du baccalauréat professionnel}

Sophie Devineau

Dix-huit mois après l'obtention de leur diplôme, confrontés au marché de l'emploi, 902 jeunes ayant un baccalauréat professionnel jugent l'école. Contrairement à l'image commune d'une jeunesse peu encline au travail, leurs propos révèlent qu'ils n'avaient pas a priori une relation négative au travail, et même c'est par l'engagement dans une formation pour un emploi qu'ils se sont sentis à l'aise dans la filière professionnelle. Dans un contexte de précarité, le travail comme valeur forte est omniprésent dans les discours. Mais la relation au travail qui se construit dans l'École, pour positive qu'elle soit, résiste mal à l'épreuve de la réalité du marché du travail lorsqu'il n'y a pas d'emploi à la clé. Le succès éducatif des bacs pro auprès des élèves et des enseignants peut être sans effet sur la difficulté de l'insertion, montrant ainsi les limites des innovations scolaires.

Mots clés

Baccalauréat professionnel, enquête, représentation de la formation, représentation du travail.

Journal of Economic Literature : I 21, J 24. 